OPEN

SUBJECT AREAS:

QUANTUM DOTS

ELECTRONIC MATERIALS

Received

13 August 2014

Accepted

16 December 2014

Published

15 January 2015

Correspondence and requests for materials should be addressed to P.R. (peter.reiss@cea.

* Current address: Lodz University of Technology,

Department of

Molecular Physics,

Zeromskiego 1 16, 90-

924 Lodz, Poland.

\section{Enhanced Charge Separation in Ternary P3HT/PCBM/CulnS 2 Nanocrystals Hybrid Solar Cells}

Aurélie Lefrançois ${ }^{1,2,3}$, Beata Luszczynska ${ }^{1,2,3,4 *}$, Brigitte Pepin-Donat ${ }^{1,2,3}$, Christian Lombard ${ }^{1,2,3}$, Benjamin Bouthinon ${ }^{4}$, Jean-Marie Verilhac ${ }^{4}$, Marina Gromova ${ }^{1,5}$, Jérôme Faure-Vincent ${ }^{1,2,3}$, Stéphanie Pouget ${ }^{1,6}$, Frédéric Chandezon ${ }^{1,2,3}$, Saïd Sadki ${ }^{1,2,3}$ \& Peter Reiss ${ }^{1,2,3}$

'Univ. Grenoble Alpes, F-38000 Grenoble, France, ${ }^{2}$ CEA-INAC-SPrAM-LEMOH, F-38000 Grenoble, France, ${ }^{3}$ CNRS UMR58 19 SPrAM, F-38000 Grenoble, France, ${ }^{4}$ CEA-Liten-DTNM, 17 rue des Martyrs, F-38000 Grenoble, France, ${ }^{5}$ CEA-INAC-SCIB-RICC, F38000 Grenoble, France, ${ }^{6}$ CEA-SP2M-SGX, F-38000 Grenoble, France.

Geminate recombination of bound polaron pairs at the donor/acceptor interface is one of the major loss mechanisms in organic bulk heterojunction solar cells. One way to overcome Coulomb attraction between opposite charge carriers and to achieve their full dissociation is the introduction of high dielectric permittivity materials such as nanoparticles of narrow band gap semiconductors. We selected $\mathrm{CuInS}_{2}$ nanocrystals of $7.4 \mathrm{~nm}$ size, which present intermediate energy levels with respect to poly(3-hexylthiophene) (P3HT) and Phenyl-C61-butyric acid methyl ester (PCBM). Efficient charge transfer from P3HT to nanocrystals takes place as evidenced by light-induced electron spin resonance. Charge transfer between nanocrystals and PCBM only occurs after replacing bulky dodecanethiol (DDT) surface ligands with shorter 1,2-ethylhexanethiol (EHT) ligands. Solar cells containing in the active layer a ternary blend of P3HT : PCBM : CuInS 2 -EHT nanocrystals in $1: 1: 0.5$ mass ratio show strongly improved short circuit current density and a higher fill factor with respect to the P3HT : PCBM reference device. Complementary measurements of the absorption properties, external quantum efficiency and charge carrier mobility indicate that enhanced charge separation in the ternary blend is at the origin of the observed behavior. The same trend is observed for blends using the glassy polymer poly(triarylamine) (PTAA).

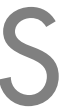

olution processed organic semiconductors and organic/inorganic hybrid materials have the potential to substantially reduce the cost of solar cells provided that the obtained power conversion efficiency (PCE) can be improved. The PCE of the best organic photovoltaic systems currently reaches around $10 \%^{1}$, while values up to $20-24 \%$ have been theoretically predicted for ideal single junction devices ${ }^{2}$. Among the limiting factors, losses related to the separation of photo-generated electron-hole pairs play an important role ${ }^{3}$. In organic solar cells the generation of free charge carriers implies the following steps $s^{4}: 1$ ) Light absorption and exciton formation in the electron-donating material ( $\pi$-conjugated polymer). The excitons are tightly bound due to the low dielectric constant of the organic absorber $\left(\varepsilon_{\mathrm{r}}=3-4\right)$ resulting in strong electrostatic interaction between opposite carriers. 2) Exciton diffusion and separation at the interface with the electron accepting material (e.g. a fullerene derivative like PCBM). 3) Formation of a Coulombically bound polaron pair (BPP) state, also called charge transfer (CT) state or exciplex state, with the electron residing on the lowest unoccupied molecular orbital (LUMO) of the acceptor and the hole residing on the highest occupied molecular orbital (HOMO) of the donor. 4) Dissociation of the BPP state and subsequent charge extraction to the electrodes. The detailed understanding of the loss mechanisms related to each of these different steps is still subject of debate 5 . While step 1 is limited by non-optimal absorption of the solar spectrum and thermalization processes, losses occurring in step 2 originate from excitons, which recombine before being able to diffuse to the donor/acceptor interface. Due to the relatively short exciton diffusion length in conjugated polymers $(\sim 10 \mathrm{~nm})$, generally the bulk heterojunction concept is applied, in which the donor and acceptor phases form an interpenetrated network ${ }^{6}$. In step 3 losses arise from geminate recombination of polaron pairs failing to fully dissociate, while in step 4 non-geminate recombination losses may occur during the charge transport to the electrode. Efficient dissociation of the BPP state into free carriers is needed to maximize the global yield of photogenerated charges and hence the device photocurrent density ${ }^{3}$. Hybrid systems composed of conjugated polymers and semiconductor nanocrystals $(\mathrm{NCs})$ are of particular interest for reducing 
the energetic driving force for charge separation due to the high dielectric permittivity and consequently low electrostatic binding energy within the inorganic phase. Nelson and coworkers explored this concept recently by combining several types of conjugated polymers with in situ formed CdS NCs ${ }^{7}$. One of the intrinsic shortcomings of binary polymer: NCs hybrids is poor, hopping-type electron transport in the inorganic phase ${ }^{8-10}$. Moreover, in order to reach the percolation threshold, high NC loading of the polymer is required ${ }^{11}$, which can lead to non-optimal morphologies and hence to reduced hole transport in the organic phase. One way to circumvent this problem is the use of NCs with dimensions spanning almost the entire thickness of the active layer. The best efficiencies have indeed been reported for large-sized nanorods or branched structures with typical arm lengths of around $100 \mathrm{~nm}^{12-14}$. Here we explore a different approach enabling enhanced free charge carrier generation in an organic photovoltaic blend through nanocrystal addition, while maintaining efficient transport. The ternary hybrid system is obtained by adding a controlled amount of $7.4 \mathrm{~nm} \mathrm{CuInS} \mathrm{nCs}_{2} \mathrm{NC}$ to a P3HT: PCBM blend. $\mathrm{CuInS}_{2}$ is a very appealing material for this study, combining a high absorption coefficient $\left(\approx 10^{5} \mathrm{~cm}^{-1}\right)$, appropriate band gap $(1.5 \mathrm{eV})$ and high dielectric constant $(\varepsilon \approx 11)$. Liao et al. recently reported a $23 \%$ increase of PCE upon the addition of a very small amount $(0.06 \mathrm{vol} \%)$ of $\mathrm{Cu}_{2} \mathrm{~S}$ or $\mathrm{CdSe} \mathrm{NCs}$ to a P3HT: PCBM blend ${ }^{15}$. This improvement has been attributed to morphological changes on the nanoscale within the hybrid thin film, as demonstrated by GISAXS and GIWAXS experiments, while electronic effects have not been considered. Here we observe the increase of PCE from $0.8 \%$ to $1.6 \%$ with respect to the P3HT: $\mathrm{PCBM}$ reference when adding a much larger amount (7 vol\%) of $\mathrm{CuInS}_{2}$ NCs. This enhancement is only observed after replacing initial dodecanethiol (DDT) surface ligands with the shorter molecule 1,2-ethylhexanethiol (EHT). We demonstrate by means of light-induced electron spin resonance, supported by optical, electrochemical and transport measurements that improved charge separation in the ternary blend is at the origin of the observed behavior. This conclusion is supported by the study of ternary hybrids using the glassy polymer poly(triarylamine) (PTAA) instead of semicrystalline P3HT, where similar trends are observed.

\section{Results}

Synthesis, functionalization and electrochemical properties of $\mathrm{CuInS}_{2}$ nanocrystals. CuInS 2 NCs showing a mean size of $7.4 \pm$ $1 \mathrm{~nm}$ and nominal composition of $\mathrm{Cu}_{0.8} \mathrm{In}_{1.1} \mathrm{~S}_{2}$ were synthesized according to the protocol published by $\mathrm{Li}$ et al. using a reaction time of 1 hour ${ }^{16,17}$. In this synthesis dodecanethiol (DDT) serves as the solvent, sulfur source and surface ligand. In order to improve charge transfer processes in the hybrid, bulky DDT ligands were replaced with 1,2-ethylhexanethiol (EHT), reducing the thickness of the insulating barrier on the nanocrystal surface from approximately $1.4 \mathrm{~nm}$ to $0.7 \mathrm{~nm}$. Furthermore, the branched ethylhexane radical provides better steric repulsion than linear chains and hence high solubility in apolar solvents. Ligand exchange was followed by ${ }^{1} \mathrm{H}-\mathrm{NMR}$ spectroscopy ( $c f$. Supplementary information), showing that the final EHT:DDT ratio was 70:30 under optimized conditions. As expected quantitative ligand exchange does not take place when replacing one alkylthiol with another. Analyzing the NMR spectra in more details reveals that DDT is bound in its deprotonated thiolate form to the nanocrystal surface, while the proton of the thiol group of EHT is still clearly visible after ligand exchange. Surprisingly the diffusion coefficients of both ligands on the nanocrystal surface are only slightly lowered with respect to the free ligands: from 1.6 to $1.05 \cdot 10^{-9} \mathrm{~m}^{2} \mathrm{~s}^{-1}$ (DDT) and from 2.1 to $1.7 \cdot 10^{-9} \mathrm{~m}^{2} \mathrm{~s}^{-1}$ (EHT). Generally a decrease of the diffusion coefficient by around one order of magnitude is observed ${ }^{18}$. One explanation could be that ligand binding occurs in a dynamic way leading to equilibrium between adsorbed and desorbed molecules. On the other hand, the observed diffusion coefficients could also reflect the mean value of tightly bound ligands in the first and more loosely bound ligands in the second coordination sphere of the $\mathrm{NCs}^{19,20}$. This argument is confirmed for DDT-capped NCs by means of thermogravimetric analysis (TGA). They exhibit a total mass loss occurring in two steps at $235-280^{\circ} \mathrm{C}$ and $400^{\circ} \mathrm{C}$ of $51 \%$, i.e. significantly higher than expected for $7.4 \mathrm{~nm} \mathrm{CuInS}{ }_{2} \mathrm{NCs}(\approx 37 \%)$. EHT-capped NCs, on the other hand, with $30 \%$ residual DDT-capping show a total mass loss of $31 \%$ occurring at $270^{\circ} \mathrm{C}$ and $400^{\circ} \mathrm{C}$, much closer to the expected value $(\approx 29 \%)$. This clearly indicates that during the ligand exchange procedure excess DDT-derived molecules (e.g. didodecyl sulfide, didodecyl disulfide) are efficiently removed from the coordination sphere of the NCs.

The reduction and oxidation potential as well as the electrochemical band gap of the NCs were determined using differential pulsed voltammetry (DPV) measurements (Suppl. Information). This technique has a higher sensitivity than cyclic voltammetry because the contribution of the capacitive current arising from the electrical double layer at the electrode is minimized. By consequence the Faradaic current corresponding to the electrolytic oxidation or reduction of the NCs is better visible. A further advantage over CV is the fact that the DPV peak is centered at the oxidation / reduction potential, which facilitates the determination of its accurate position $^{20}$. However, for DDT-capped NCs no clear signal could be obtained in measurements of the first oxidation potential, probably due to the comparably thick organic ligand shell. After ligand exchange with EHT, the ionization potential (IP) and electron affinity (EA) have been determined using the ferrocene/ferrocenium redox couple as standard $\left(\mathrm{Fc} / \mathrm{Fc}^{+}:-4.8 \mathrm{eV}\right)^{21}$ to be -5.6 and $-4.1 \mathrm{eV}$, respectively, resulting in an electrochemical band gap of $1.5 \mathrm{eV}$. Taking into account the IP and EA values of regioregular P3HT $(-4.9 \mathrm{eV} /-3.0 \mathrm{eV})$ and PCBM $(-6.4 \mathrm{eV} /-4.3 \mathrm{eV})^{22}$, the studied $\mathrm{CuInS}_{2}$ NCs provide additional intermediate energy levels enabling cascade-like charge transfer ${ }^{23}$. The energy levels and electronic processes occurring in the ternary hybrid are summarized in Fig. 1.

Light-induced electron spin resonance. LESR detects photogenerated paramagnetic species such as polarons by inducing transitions between energy levels splitting up in an external magnetic field. Due to the long lifetime of the charge-separated state in organic D-A blends, this method is well suited for studying the formation of radical cations like $\mathrm{P}_{3} \mathrm{HT}^{+\circ}$ or radical anions like $\mathrm{PCBM}^{-}$. The corresponding ESR signals are situated at

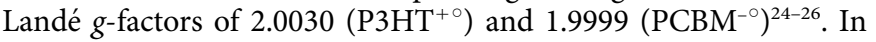
conjugated polymer/NCs hybrid blends no signals corresponding to radicals generated in the nanocrystal phase have been reported ${ }^{27,28}$. Signal broadening and/or localization at $g$-factors strongly shifted from the organic species are possible causes. First, we study the LESR data of binary blends containing PCBM or P3HT and DDTcapped NCs. In the case of the PCBM : NCs-DDT blend, no signal of the $\mathrm{PCBM}^{-}$radical anion is detectable in the dark and only a very weak signal appears under 473-nm illumination (Fig. 2a). As both components absorb at this wavelength, the absence of the LESR signal indicates that no photo-induced charge transfer leading to long-lived carriers between PCBM and $\mathrm{CuInS}_{2}-\mathrm{DDT}$ NCs takes place. In the NCs blend with P3HT, an ESR signal is already observed in the dark (Fig. 2b) with a $g$-factor corresponding to $\mathrm{P}_{3} \mathrm{HT}^{+\circ}$. It can be attributed either to intrinsic defects in $\mathrm{P} 3 \mathrm{HT}$, to doping occurring during air exposure ${ }^{29}$ or to paramagnetic species resulting from 'parasitic' light exposure of the blend. Since under illumination no change in signal intensity is observed, the latter assumption is clearly not valid. These measurements have been carried out under conditions maximizing the signal in the dark (in phase). When measuring out of phase, a signal can be detected at a 


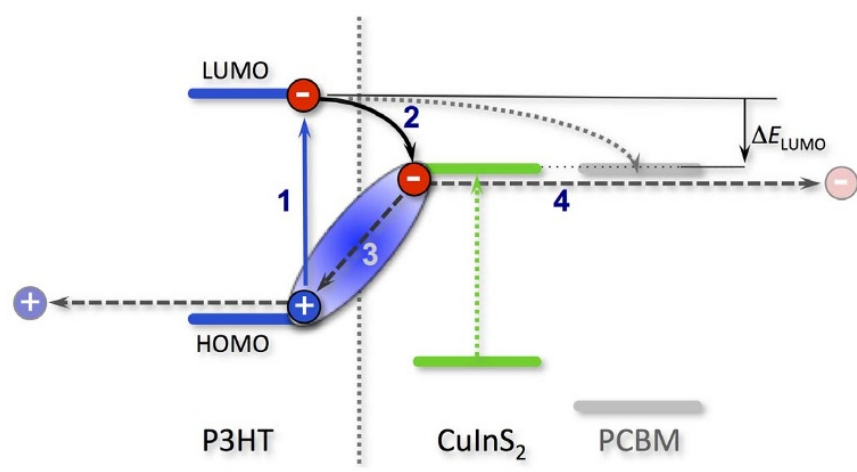

Figure 1 Energy level diagram of the P3HT: PCBM: $\mathrm{CuInS}_{2} \mathrm{NCs}$ hybrid, illustrating electronic processes possible at the $\mathrm{P} 3 \mathrm{HT} / \mathrm{NCs}$ interface upon light absorption by the polymer: 1) exciton formation and diffusion to the D/A interface; 2) charge separation and formation of a bound polaron pair (BPP) state; 3) geminate recombination or 4) full dissociation of the BPP state leading to free carriers. The energetic driving force for the dissociation of the BPP state scales with the LUMO levels offset $\Delta E_{\mathrm{LUMO}}$. Charge transfer to PCBM can take place both from the polymer and the NCs.

magnetic field corresponding to the $g$-factor of $\mathrm{P} 3 \mathrm{HT}^{+\circ}$. This signal immediately disappears when the light is turned off, and the light-on / light-off behavior can be reproduced many times in a completely reversible way. Consequently, the signal can be unambiguously

a)

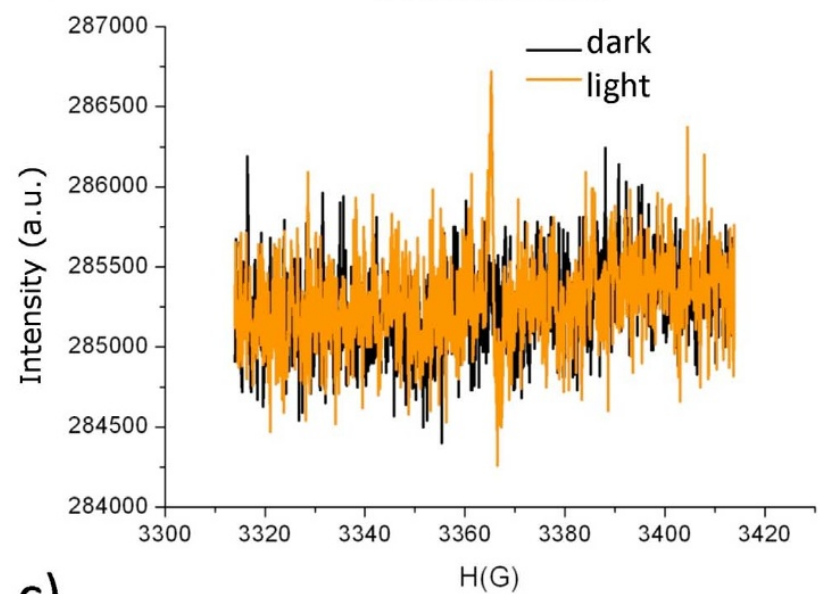

c)

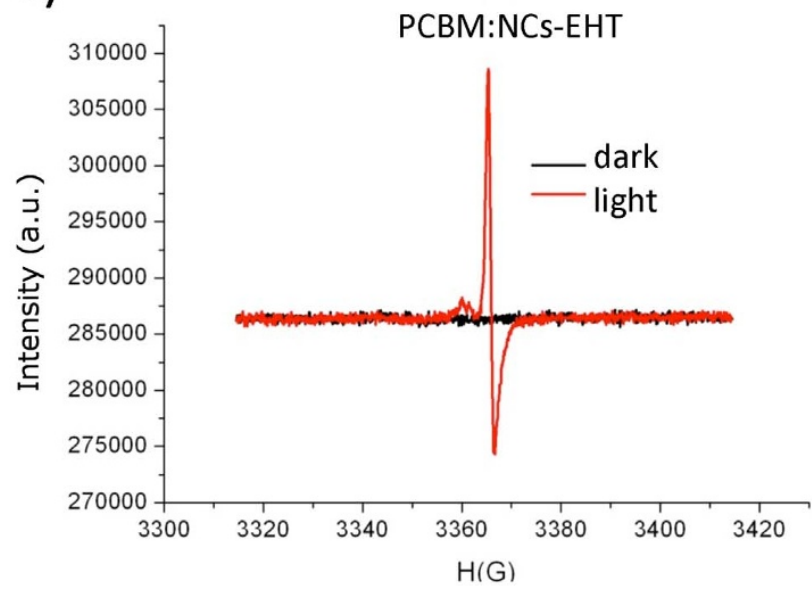

ascribed to a photo-induced phenomenon, whose origin is under current investigation. We note that no 'out-of-phase' signal is detected upon illumination of P3HT/NCs-EHT blends. For PCBM or P3HT blended with EHT-capped NCs a clear light effect is observed. More precisely, under illumination a distinct signal appears in the case of PCBM:NCs-EHT, while a strong increase of the signal with respect to the dark is detected in the case of P3HT: NCs-EHT (Fig. 2c/d). This result clearly illustrates the crucial role of the different steric hindrance of the NCs capping ligands (EHT vs. DDT). When going to the ternary blends, extracting the influence of NCs on the LESR behavior is less straightforward: signal intensities of the different samples cannot be directly compared and eventual interactions between the spins arising from the radical cation and radical anion could be expected ${ }^{26}$. Fig. 3 shows the comparison of the LESR spectra of the ternary blends containing DDT- or EHT-capped NCs, P3HT and PCBM.

Both the additions of DDT- and EHT-capped NCs have similar effects on the LESR data. First, we qualitatively observe that the $\mathrm{P}_{3} \mathrm{HT}^{+\circ} / \mathrm{PCBM}^{-\circ}$ signal intensity ratio increases significantly. This effect is more pronounced with EHT- than with DDT-capping. Table 1 gives the peak intensity ratios $\mathrm{P} 3 \mathrm{HT}^{+\circ} / \mathrm{PCBM}^{-\circ}$ recorded under illumination and after 6 minutes of relaxation in the dark. We stress that the absolute values are meaningless and that only their comparison can be exploited. In case of the binary blend PCBM : P3HT, we get an intensity ratio equal to 1.0 , which increases up to 1.7 for P3HT: $\mathrm{PCBM}:$ NCs-DDT and to 2.5 for P3HT: PCBM:NCs-EHT. The first hypothesis is that this increase

b)

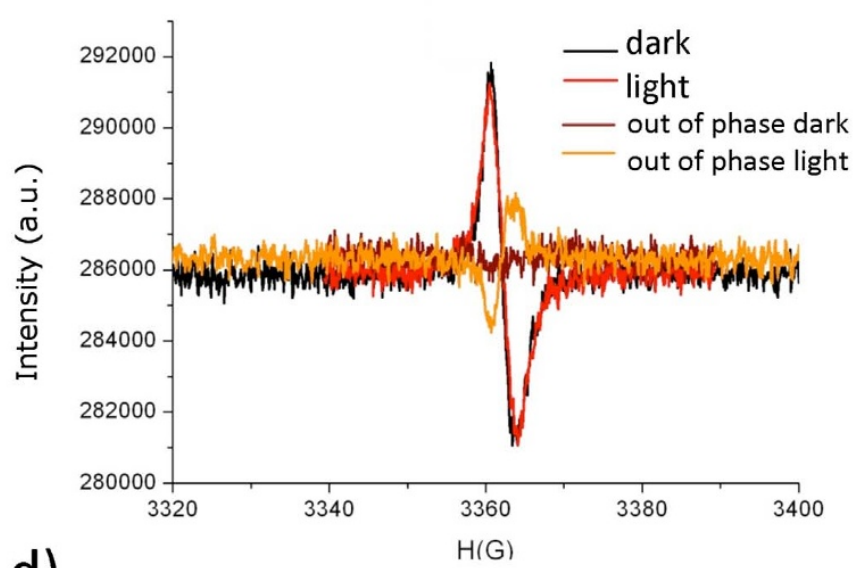

d)

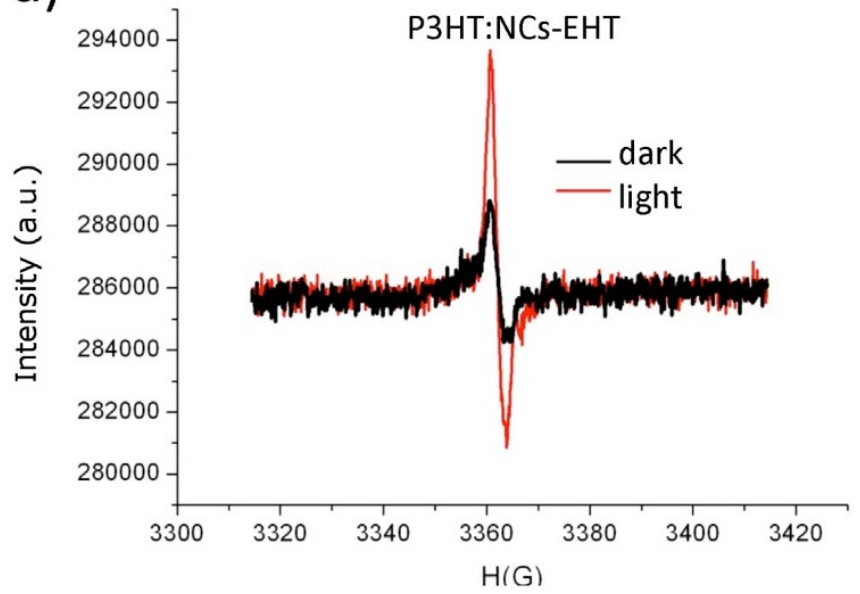

Figure $2 \mid$ LESR spectra of thin films of binary blends containing DDT- or EHT-capped NCs and PCBM (a,c) or P3HT (b,d), recorded in the dark or under $473-\mathrm{nm}$ illumination $\left(P_{\text {microwave }}=20 \mathrm{~dB}(100 \mu \mathrm{W}), P_{\text {laser }}=22 \mathrm{~mW}\right)$. 


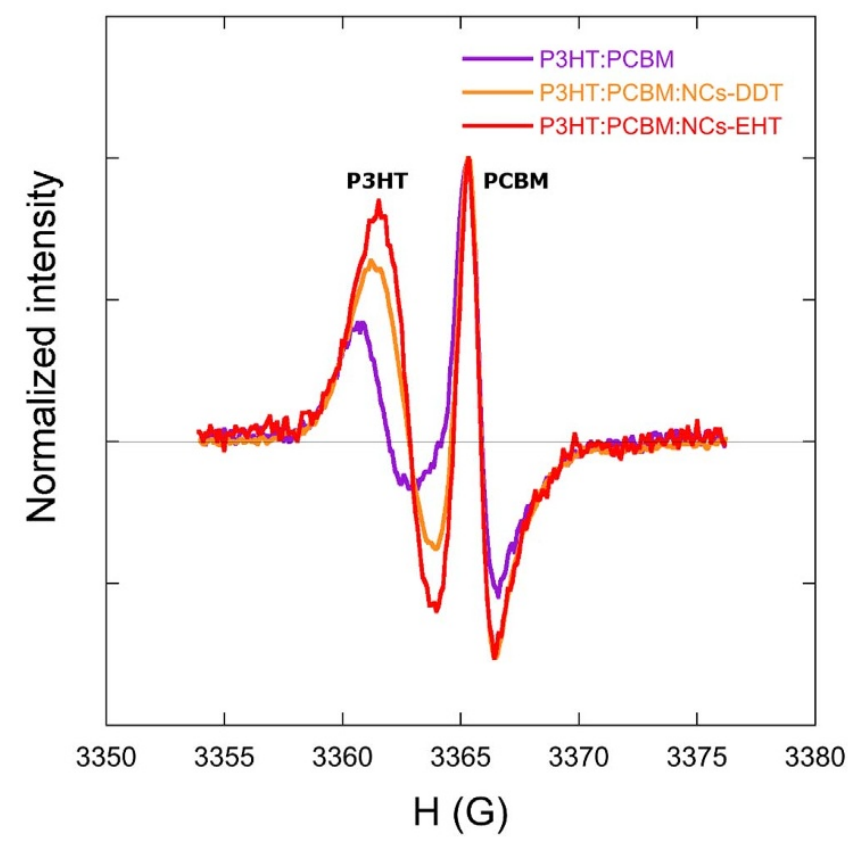

Figure 3 LESR spectra of the binary and ternary hybrid films recorded at $20 \mathrm{~K}$ under $473 \mathrm{~nm}$ illumination $\left(P_{\text {microwave }}=20 \mathrm{~dB}(100 \mu \mathrm{W}), P_{\text {laser }}=\right.$ $22 \mathrm{~mW}$; mass ratios $\mathrm{P} 3 \mathrm{HT}: \mathrm{PCBM}=1: 1$ and $\mathrm{P} 3 \mathrm{HT}: \mathrm{PCBM}: \mathrm{NCs}=$ $1: 1: 0.5$, respectively). The spectra obtained for the same films in the dark have been subtracted and the intensity has been normalized using the signal of PCBM.

is due to additional electronic transfers between P3HT and NCs. For EHT-capped NCs, this assumption is coherent with the observed illumination effect on the spectrum of the binary blend P3HT: NCs-EHT (Fig. 2d). However, in the case of DDT-capped NCs blended with P3HT we do not observe any light effect on the ESR spectrum (Fig. 2b). One explanation might be a change in morphology in the ternary blends leading to enhanced charge transfer between P3HT and NCs. The low temperature relaxation of the LESR signals shows two components - a fast one, leading to a direct signal decrease after switching off the irradiation, and a slower one on the scale of hours, during which the remaining signal vanishes (cf. Suppl. Information). Heinemann et al. observed similar relaxation kinetics in P3HT : CdSe NCs blends ${ }^{24}$. The fast decay has been attributed to bimolecular (i.e. non-geminate) recombination and the persistent signal to charge capture in deep trap states generated on the polymer due to morphology changes induced by nanocrystal addition. In the ternary hybrids of the present study, both the signals related to $\mathrm{P}_{3} \mathrm{HT}^{+\circ}$ and $\mathrm{PCBM}^{-\circ}$ are more persistent than in $\mathrm{P} 3 \mathrm{HT}: \mathrm{PCBM}$ alone. Moreover, the fraction of persistent charges is much higher in the case of the P3HT: CdSe hybrids ${ }^{24}$ than in the ternary hybrids investigated here. When comparing the relative signal intensities six minutes after switching off the light, we observe distinct differences in relaxation behavior (Table 1). For P3HT : PCBM a strong decrease of both signals to around $20 \%$ of their initial values takes place. In the ternary hybrids, the signal decrease is less pronounced, in particular for $\mathrm{PCBM}^{-\circ}$ showing still $50-60 \%$ of the initial intensity. Slower relaxation kinetics can be ascribed to changes in the charge recombination dynamics and/or to morphological changes. The faster decay observed for both signals in case of EHT-capped NCs shows that the shorter ligand facilitates charge recombination processes. Importantly, the relaxation data has been acquired at low temperature $(20 \mathrm{~K})$ and in the absence of specific contacts at the hybrid blend for electron and hole extraction. In order to address the question whether the photogenerated charges are mobile at room temperature and can be efficiently used in photovoltaic devices, we integrated the hybrids into solar cells, using identical samples and film processing conditions as for the LESR measurements.

Solar cell measurements. Table 2 gives an overview of the different mass fractions used for the preparation of the hybrid blends, and of the calculated volume fractions occupied by NCs and PCBM in the polymer matrix. The blends were deposited by spin-coating on ITOcoated glass substrates containing a 30-40 nm thick layer of PEDOT:PSS. The spinning parameters have been adjusted for obtaining $\mathrm{a} \approx 200$-nm thick photoactive layer. Finally $80-100 \mathrm{~nm}$ thick Al electrodes were thermally evaporated on top, and the devices were encapsulated with glass covers. Figure $4 \mathrm{a}$ presents the $J(V)$ characteristics of the devices containing different mass fractions of NCs under irradiation with a solar simulator (AM1.5, one sun). As can be seen from Table 3, the same trends are observed before and after annealing at $120^{\circ} \mathrm{C}$ for $15 \mathrm{~min}$ : the ternary hybrid solar cell containing EHT-capped NCs outperforms the cell containing DDT-capped NCs, and the binary P3HT:PCBM device shows intermediate values. The most important differences between the device with EHT-capped NCs and the device without NCs lie in the short circuit current density and in the fill factor. The power conversion efficiency of the P3HT: $\mathrm{PCBM}$ reference cell $(0.8 \%)$ is low compared to the best literature results $(4-5 \%)^{30}$, yet consistent with reported values of non optimized test cells ${ }^{31}$. Non-optimal deposition conditions, such as fast film growth rate without control of the solvent evaporation speed ${ }^{32}$ as well as the absence of a $\mathrm{LiF}$ layer at the active layer/Al electrode interface are possible origins of this lower performance. Batch-to-batch variations in the polymer purity and macromolecular parameters also influence the device performances. However, the three sets of devices allow for the direct comparison of the binary and ternary blends prepared under identical processing conditions. Taking into account the results from the LESR measurements, the $J(V)$ characteristics can be interpreted as follows: In both hybrid blends $\mathrm{CuInS}_{2} \mathrm{NCs}$ add additional channels for charge transfer from P3HT. Efficient transfer is possible even in the case of bulky DDT ligands, confirming the results of Kruszynska et al. ${ }^{33}$ who studied blends of P3HT and trioctylphosphine oxide / DDT-capped CuInS $\mathrm{S}_{2} \mathrm{NCs}$ by means of photo-induced absorption. We hypothesize that hydrophobic interactions between the alkyl chains of the ligands and of the polymer enable the close proximity between the nanocrystal surface and the polymer backbone. Such hydrophobic interactions are not expected between NCs and PCBM, inhibiting efficient charge transfer in that case. As the volume fraction of NCs in the hybrid is far below the percolation threshold, the extraction of charges generated on the NCs and their transport to the Al electrode is inefficient, leading to low photocurrent and PCE observed in the P3HT : PCBM : NCs-DDT hybrid. The inflection point in the $J(V)$

Table 1 | LESR peak intensity ratio $\mathrm{P} \mathrm{HT}^{+\circ} / \mathrm{PCBM}^{-\circ}$ measured for the different blends and intensity (peak-peak) $I_{(6 \text { min) }}$ after 6 min relaxation time at $20 \mathrm{~K}$ with respect to the initial intensity $\mathrm{I}_{0}$ for $\mathrm{P} \mathrm{HT}^{+\circ}$ and $\mathrm{PCBM}^{-\circ}$

Composition of the blend

P3HT: PCBM

P3HT: PCBM: NCs DDT

P3HT: PCBM: NCs EHT
Intensity ratio $\mathrm{P} \mathrm{HT}^{+\circ} / \mathrm{PCBM}^{-\circ}$

1.0

1.7

2.5
$I_{(6 \mathrm{~min})} / I_{0} \mathrm{P} 3 \mathrm{HT}^{+0}$

0.2

0.4

0.3
$I_{(6 \mathrm{~min})} / I_{0} \mathrm{PCBM}^{-\circ}$

0.2

0.6

0.5 
Table 2 | Mass and volume fractions of PCBM and $\mathrm{CuInS}_{2} \mathrm{NCs}$ in P3HT. For the calculation of the volume fractions the following densities have been used: P3HT $-1.1 \mathrm{~g} / \mathrm{cm}^{3}$, PCBM $-1.72 \mathrm{~g} / \mathrm{cm}^{3}, \mathrm{CulnS}_{2}-4.75 \mathrm{~g} / \mathrm{cm}^{3}$

\begin{tabular}{lcccc}
$m_{\mathrm{P} 3 \mathrm{H}}: m_{\mathrm{PCBM}}: m_{\mathrm{NCs}}$ & Mass fraction of $\mathrm{NCs}$ & Volume fraction of $\mathrm{NCs}$ & Mass fraction of PCBM & Volume fraction of PCBM \\
\hline $1: 1: 0$ & $0 \%$ & $0 \%$ & $50 \%$ & $39 \%$ \\
$1: 1: 0.5$ & $20 \%$ & $7 \%$ & $40 \%$ & $36 \%$ \\
$1: 1: 1$ & $33.33 \%$ & $12.4 \%$ & $33.33 \%$ & $34.2 \%$ \\
\hline
\end{tabular}

curve of this hybrid observed in Fig. 4a, resulting in a significantly lower $V_{o c}$ than for the other two blends, indicates electric field dependent generation of free carriers. With increasing voltage, the operating conditions of the device shift from short-circuit to opencircuit and the electric field due to electrode charge approaches zero (flat band conditions). This reduction of internal field leads to an increase of the steady state charge carrier density in the active layer. By consequence the probability of non-geminate recombination increases, resulting in the observed $J(V)$ behavior. In the case of EHT-capped NCs, electron transfer to PCBM is possible (cf. Fig. 2c), improving charge extraction to the electrode in this case. NC induced morphological changes in the organic phase have been shown to influence the $J / V$ characteristics ${ }^{15}$ but can be ruled out here as dominating factor, based on the differences observed for EHTand DDT-capped NCs.

In order to investigate the influence of the NCs on the spectral response of the solar cells, we carried out external quantum efficiency (EQE) measurements (Fig. $4 \mathrm{~b}$ ). The spectrum of the P3HT: PCBM thin film without NCs shows an average EQE value of $25 \%$ in the spectral range of $350-630 \mathrm{~nm}$. The shape and spectral range is in good agreement with the data reported for fast grown P3HT : PCBM films ${ }^{32}$. The same applies for the device containing DDT-capped NCs, which however shows a much lower EQE with a maximum of $18 \%$. The device containing EHT-capped NCs exhibits a maximum efficiency of $50 \%$ at $500 \mathrm{~nm}$. It also shows a decreased contribution of PCBM (300-400 nm range) to EQE compared to the other two blends. As seen in the UV-vis spectra (Suppl. Information, Fig. S5), adding 7 vol.\% of NCs into the P3HT: PCBM blend ( $1: 1: 0.5$ mass ratio) does not substantially modify the light harvesting properties of the obtained thin film or solar cell. NCs show mainly absorption in the UV and visible range whereas their absorbance in the near infrared range is very low. Both the pure P3HT: PCBM and the NCs containing thin films display vibronic structure with a clearly resolved lower energy shoulder. This behavior is expected, as semicrystalline P3HT shows less ground state conformational disorder compared to the polymer in solution, where only a featureless broad absorption peak is visible ${ }^{34}$. We emphasize that for the investigated film thickness and NC concentrations no distinct differences between the absorption features of binary P3HT : $\mathrm{PCBM}$ and ternary P3HT: PCBM : NCs blends could be detected.

Finally, we determined charge carrier mobilities in the devices by means of photo-CELIV (charge carrier extraction by linearly increasing voltage) measurements. In these experiments a nanosecond $532 \mathrm{~nm}$ laser pulse is directed on the transparent ITO electrode. Transit times of the photogenerated carriers have been determined for different electric field strengths and mobilities have been calculated using the following relationship ${ }^{35}$ :

$$
\mu=\frac{2 d^{2}}{3 A t_{\max }^{2}\left[1+0.36 \frac{\Delta j}{j(\mathrm{o})}\right]}
$$

with $d$ being the film thickness, $A$ the voltage rise speed $d U / d t$ in $\mathrm{Vs}^{-1}, t_{\max }$ the delay after the light pulse when the extracted current reaches its maximum, $j$ and $j(0)$ the current generated by the mobile charges and the capacitive current arising from the voltage ramp. Eq. 1 is valid for systems showing moderate conductivity, where $j$ and $j(0)$ are of the same order of magnitude. The CELIV technique probes the mobility of the more mobile charge carriers, corresponding to electrons in our case ${ }^{36}$. In the present configuration illumination of the devices occurs through the ITO electrode, where the negative bias is applied. For all the experiments, the delay between the laser pulse and the voltage ramp was set to $550 \mathrm{~ns}$. The laser output power of $50 \mu \mathrm{J} / \mathrm{cm}^{2}$ was lowered by means of optical densities to approx. $50 \mathrm{~nJ} / \mathrm{cm}^{2}$ on the sample, with the goal to keep the photogenerated carrier concentration lower than $10^{17} \mathrm{~cm}^{-3}$. Higher carrier concentrations can lead to wrong estimations of the mobility, due to charge recombination processes and space charge effects ${ }^{37}$. For all samples, the device area was chosen in a way that the RC time constant be kept below 300 ns (the extracted photo-CELIV current reaches its maximum at a time scale larger than $1 \mu \mathrm{s})$. Fig. 5 a shows the comparison of the mobility values obtained for the ternary blends containing either DDT- or EHT-capped NCs before thermal annealing. The extracted mobilities are $2.6 \cdot 10^{-5} \mathrm{~cm}^{2} \cdot \mathrm{V}^{-1} \mathrm{~s}^{-1}$ (DDT) and
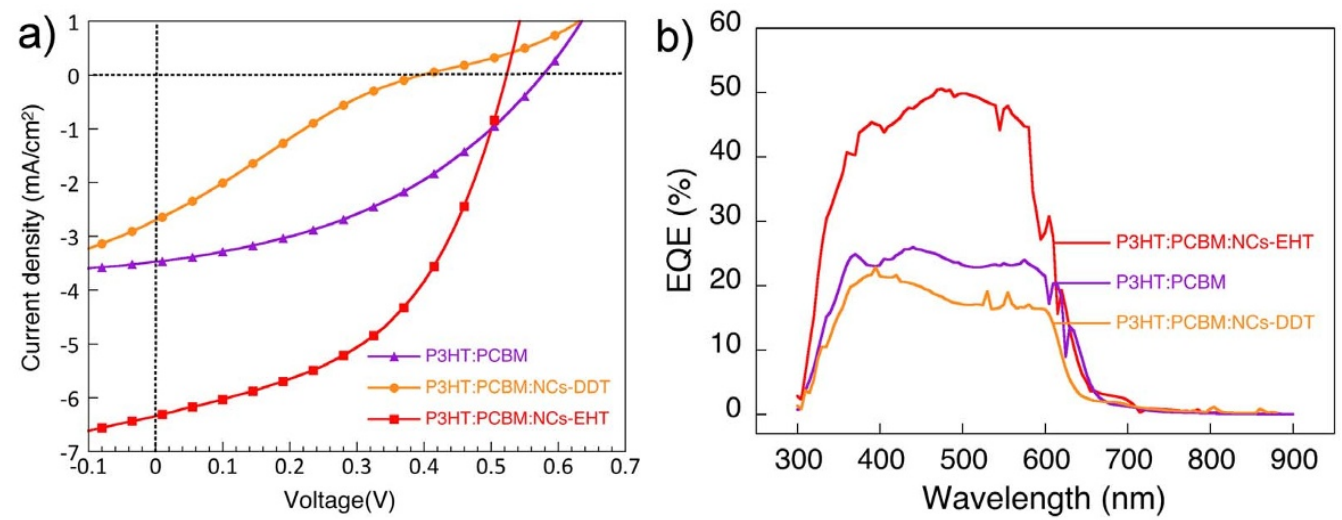

Figure $4 \mid$ (a) $J(V)$ curves obtained under simulated solar light (AM1.5 one sun conditions) of solar cells containing ternary blends of P3HT, PCBM and DDT- or EHT-capped CuInS 2 NCs (1:1:0.5 mass ratio) after 15 min annealing at $120^{\circ} \mathrm{C}$. For comparison the curve obtained with an identical solar cell containing the binary P3HT: PCBM mixture (1:1 mass ratio) is shown. (b) External quantum efficiency spectra of the different solar cells measured under identical illumination conditions. 
Table 3 | Solar cell characteristics measured under AM1.5 one sun conditions after annealing for 15 min at $120^{\circ} \mathrm{C}$ in argon atmosphere; values in brackets: before annealing

\begin{tabular}{|c|c|c|c|c|}
\hline & $\mathrm{FF}$ & $V_{o c}(V)$ & $\mathrm{J}_{\mathrm{sc}}\left(\mathrm{mA} / \mathrm{cm}^{2}\right)$ & Efficiency (\%) \\
\hline $\begin{array}{l}\text { P3HT : PCBM } 1: 1 \\
\text { P3HT : PCBM: NCS-DDT } 1: 1: 0.5 \\
\text { P3HT: PCBM:NCs-EHT } 1: 1: 0.5 \\
\text { P3HT : PCBM:NCs-EHT } 1: 1: 1\end{array}$ & $\begin{array}{l}0.40(0.43) \\
0.23(0.27) \\
\mathbf{0 . 4 8}(0.39) \\
0.37(0.36)\end{array}$ & $\begin{array}{l}0.58(0.33) \\
0.40(0.23) \\
\mathbf{0 . 5 2}(0.30) \\
0.44(0.32)\end{array}$ & $\begin{array}{r}-3.47(2.00) \\
-2.70(0.07) \\
-6.34(4.05) \\
2.19(1.79)\end{array}$ & $\begin{array}{l}0.81(0.29) \\
0.24(0.06) \\
1.60(0.47) \\
0.35(0.20)\end{array}$ \\
\hline
\end{tabular}

$6.1 \cdot 10^{-5} \mathrm{~cm}^{2} \cdot \mathrm{V}^{-1} \mathrm{~s}^{-1}(\mathrm{EHT})$ at $\mathrm{E}^{1 / 2}=280 \mathrm{~V}^{1 / 2} \cdot \mathrm{cm}^{-1 / 2}$; hence ligand exchange leads to a 2.5 -fold increase in electron mobility in the hybrid thin films. At room temperature, both ternary blends containing DDT- or EHT-capped NCs show similar positive electric field dependence of mobility. This stands out against the negative field dependence reported for pure P3HT films ${ }^{11,38}$. Additional measurements at variable temperature are necessary to investigate the origin of this trend. Fig. $5 \mathrm{~b}$ shows photo-CELIV measurements on the ternary blend containing EHT-capped NCs after annealing and compares them to the binary P3HT: PCBM blend. For all field strengths, the mobility of the ternary device $\left(4.7 \cdot 10^{-4} \mathrm{~cm}^{2} \cdot \mathrm{V}^{-1} \mathrm{~s}^{-1}\right.$ at $\left.280 \mathrm{~V}^{1 / 2} \cdot \mathrm{cm}^{-1 / 2}\right)$ is slightly lower than that of the binary P3HT: PCBM device $\left(7.0 \cdot 10^{-4} \mathrm{~cm}^{2} \cdot \mathrm{V}^{-1} \mathrm{~s}^{-1}\right)$. It is therefore safe to conclude that nanocrystal addition does not lead to an increase of charge mobility in the hybrid films.

Semicrystalline polymers like $\mathrm{P} 3 \mathrm{HT}$ are very sensitive to the processing and annealing conditions, and slight modifications in the thin film morphology can strongly influence the solar cell performance $^{34}$. In order to evaluate the influence of NC addition to an amorphous polymer matrix, we investigated hybrids using poly (triarylamine) (PTAA). As compared to P3HT, PTAA shows a higher LUMO level offset and similar HOMO level offset with the electron acceptors (NCs and PCBM) present in the blends ( $c f$. Suppl. Information Fig. S7). Therefore in both cases the same charge transfer processes are enabled from the energetic point of view. The UVvis absorption spectra of solar cells containing PTAA: PCBM:NCs hybrids using $1: 1: 0,1: 1: 0.25,1: 1: 0.5$ and $1: 1: 1$ mass fractions can be fully superposed (Fig. S9). Confirming the aforementioned observations with $\mathrm{P} 3 \mathrm{HT}$, CuInS 2 NCs added in comparably low concentration to the polymer:PCBM blend do not modify the absorption properties of the hybrid thin films. By consequence the light harvesting features of the PTAA hybrids are limited to the UV/ blue range, as the absorption onset of the polymer is located at around $410 \mathrm{~nm}$ (band gap $2.95 \mathrm{eV}$ ) ${ }^{39}$. Using $1: 1: 0.25$ and $1: 1: 0.5$ ratios results in a strong increase of the short circuit current density of hybrid solar cells as compared to PTAA : PCBM binary devices (Fig. S8, Table S1). While the absolute values of $J_{S C}$ and PCE are much lower than in the case of P3HT, the same trend in terms of optimum NC concentration is observed and the 1:1:0.5 blend shows the best performance.

\section{Discussion}

Using $20 \mathrm{wt} \%$ (7 vol\%) of EHT-capped $\mathrm{CuInS}_{2}$ NCs in a ternary P3HT: PCBM: NCs hybrid strongly influences the solar cell characteristics as compared to the reference cell P3HT: PCBM. Mainly the short circuit current density and fill factor are affected, resulting in increased power conversion efficiency. At the same time UV-vis absorption and EQE measurements on the hybrid ternary blend do not reveal significant changes in the light harvesting properties with respect to the binary blend. Higher charge carrier mobilities can also be ruled out to explain the improved solar cell characteristics, as shown by photo-CELIV measurements. Consequently, our results are in stark contrast with reported binary and ternary systems containing small fractions of NCs. There improved charge transport properties have been observed, which were most often attributed to changes in the polymer chain packing ${ }^{15,40,41}$. The function principle of the hybrid system also differs from recently developed fully organic ternary photovoltaic blends. They aim at an extension of the spectral range of light absorption by adding different types of sensitizers (dyes, polymers, small molecules) to the organic blend ${ }^{42,43}$. To give an example, Huang et al. added $1 \mathrm{wt} \%$ of a near-infrared absorbing squaraine dye to a P3HT: $\mathrm{PCBM}$ blend, resulting in a $38 \%$ increase of PCE up to a value of $4.5 \%{ }^{44}$. The synergistic action of Förster resonant energy transfer (FRET) and a change in the film morphology has been proposed to be at the origin of this improvement. In the present study, LESR experiments show a marked influence on the
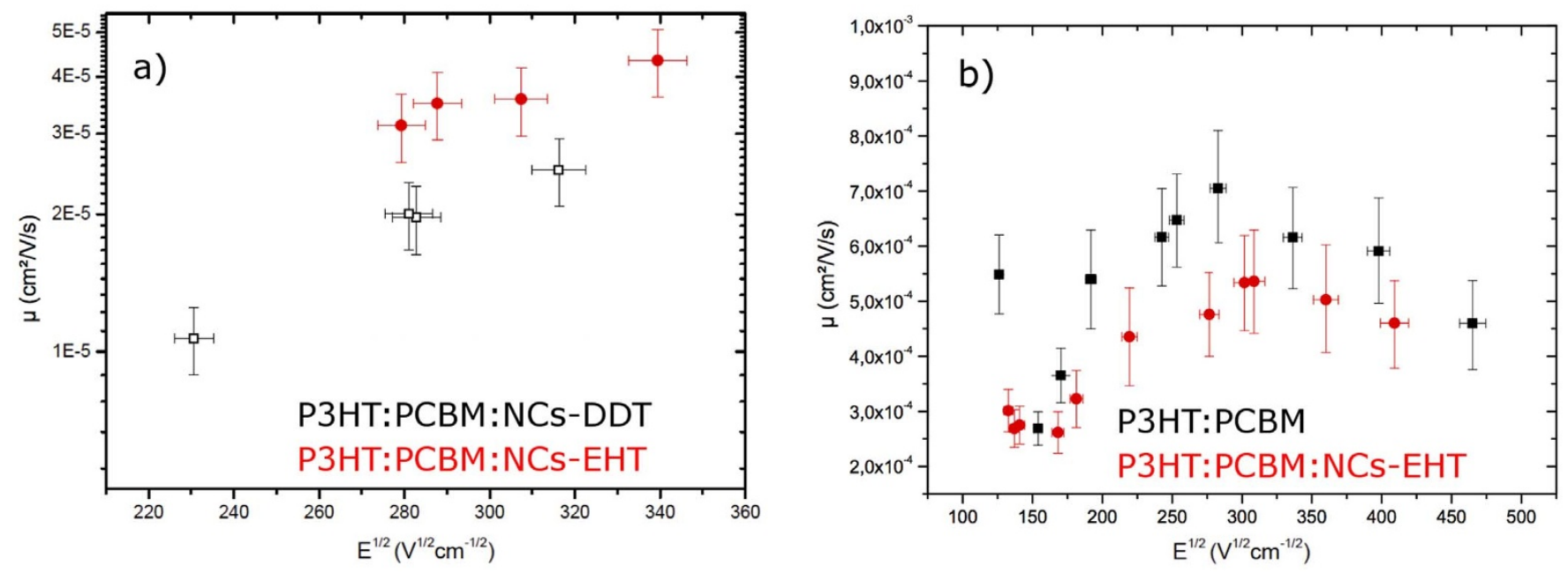

Figure 5 Electric field dependence of the electron mobility measured at room temperature. Error bars take account for the incertitude in the determination of $t_{\max }$ and of the active layer thickness. (a) Comparison of ternary blends containing DDT-and EHT-capped NCs. (b) Comparison of the ternary blend containing EHT-capped NCs with the binary P3HT : PCBM blend after annealing for 15 min to $120^{\circ} \mathrm{C}$. 
charge transfer processes taking place upon addition of NCs to the P3HT : PCBM blend. In the case of short EHT ligands, electron transfer from the polymer to NCs and, to a lower extent, from NCs to PCBM has been unambiguously demonstrated. High dielectric constant inorganic NCs contribute thus strongly to the efficient separation of the BPP state; the dielectric permittivities of the materials are 3.0 (P3HT), 3.9 (PCBM) and $11\left(\mathrm{CuInS}_{2}\right)$. Small volume fractions of NCs preferably localize in amorphous, low mobility domains of P3HT as has been shown in the case of P3HT: CdSe NCs hybrids obtained by directional epitaxial crystallization ${ }^{45}$. While such domains generally act as dead-ends for charge transport, filling them with NCs gives rise to an improved extraction of photogenerated charge carriers. As a consequence of the intermediate energy levels of the chosen $\mathrm{CuInS}_{2}$ EHT NCs they can act as effective electron relays between P3HT and PCBM clusters. The same energy level alignment with larger LUMO level offset exists in hybrid blends based on PTAA. Using this amorphous polymer results in a similar trend in terms of short circuit current density $v s$. NC concentration as in the case of semicrystalline P3HT, corroborating the proposed role of NCs in ternary hybrid blends. We anticipate that the same behavior will be observable for other polymers, as long as an appropriate energy level alignment exists. The presented results underpin the relevance of applying semiconductor NCs in OPV blends, highlighting their beneficial role in charge separation without negatively impacting charge transport when used in low concentration. Further improvement of the absolute solar cell efficiencies is expected through the optimization of device fabrication parameters (e.g. active layer thickness, interface materials), nanocrystal surface chemistry and the use of alternative conjugated polymers ${ }^{22}$.

\section{Methods}

Chemicals. Copper(I) iodide ( $>99.9 \%)$, indium(III) acetate $(>99.9 \%)$, dodecanethiol (99\%), 1,2-ethylhexanethiol (99\%), ITO substrates, PEDOT : PSS (Baytron $\mathrm{PH}$ ), poly(bis(4-phenyl)(2,4,6-trimethylphenyl)amine (PTAA; $\mathrm{M}_{\mathrm{n}}=9,000$; $\left.\mathrm{M}_{\mathrm{w}}=19,000\right)$ and anhydrous solvents were purchased from Sigma-Aldrich. Regioregular P3HT (Merck SP001, $\mathrm{M}_{\mathrm{n}}=24,000 ; \mathrm{M}_{\mathrm{w}}: 55,000 ; \mathrm{RR}=95 \%$ ) was purchased from Merck; PCBM from Nano-C.

Synthesis of 7.4-nm CuInS $\mathbf{N C s}^{17}$. CuInS ${ }_{2}$ nanocrystals were synthesized by reacting copper(I) iodide (190 $\mathrm{mg}, 1 \mathrm{mmol}$ ) with indium(III) acetate $(292 \mathrm{mg}$, $1 \mathrm{mmol}$ ) in dodecanethiol $(5 \mathrm{~mL})$ using a $50 \mathrm{~mL}$ three neck flask equipped with a condenser. First the reaction mixture was degassed for 15 min under primary vacuum using a Schlenk line. Under argon atmosphere the temperature was then raised to $100^{\circ} \mathrm{C}$ at $10^{\circ} \mathrm{C} / \mathrm{min}$. The mixture was stirred for one hour before degassing once again for $1 \mathrm{~min}$ and backfilling with argon, yielding a yellow, transparent solution. Stirring was continued and the temperature was raised to $230^{\circ} \mathrm{C}$ at $30^{\circ} \mathrm{C} /$ min, resulting in a colour change from yellow over orange and red to dark brown. The mixture was left for one hour at this temperature. After cooling to room temperature, the nanocrystals were purified by adding $5 \mathrm{~mL}$ of methanol and centrifugation at $5000 \mathrm{rpm}$ for $5 \mathrm{~min}$. A second cycle or purification was carried out by adding $10 \mathrm{~mL}$ of chloroform to the precipitate, followed by $5 \mathrm{~mL}$ of methanol and centrifugation as before. The precipitate was finally solubilized in $10 \mathrm{~mL}$ of chloroform or toluene.

Ligand exchange. $200 \mu \mathrm{L}$ of 1,2-ethylhexanethiol were added to a colloidal solution of $\mathrm{CuInS}_{2}$ nanocrystals ( $3 \mathrm{~mL}$ in $\mathrm{CHCl}_{3}$, concentration: $100 \mathrm{mg} / \mathrm{mL}$ ) and stirred under argon atmosphere and reflux for four hours, then at room temperature overnight. The nanocrystals were precipitated by adding a mixture of $10 \mathrm{~mL}$ of ethanol and $20 \mathrm{~mL}$ of $\mathrm{CHCl}_{3}$.

Materials Characterization. The obtained NCs and thin films have been analysed using a JEOL 4000EX High Resolution Transmission Electron Microscope (HRTEM) operated at $400 \mathrm{kV}$. UV-visible spectroscopy has been performed with a HEWLETT PACKARD 8452A diode array spectrophotometer. X-ray diffraction measurements were carried out in the Bragg-Brentano reflection geometry using a Panalytical Empyrean diffractometer provided with $\mathrm{Cu} \mathrm{K} \alpha$ radiation, $\lambda=1.5418 \AA$.

Electrochemical measurements. Voltammetric measurements were performed under Argon atmosphere inside a glove box using an Autolab3 potentiostat/ galvanostat. Potentials were recorded versus an Ag wire pseudo reference electrode using Pt counter electrode and work electrode (diameter: $4 \mathrm{~mm}$ ). The samples were prepared by drop casting $10 \mu \mathrm{L}$ of a $20 \mathrm{mg} \cdot \mathrm{mL}^{-1}$ nanocrystals' colloidal solution in chloroform on the working electrode and subsequent immersion of the electrode in the ionic liquid. DPV curves for the free ligands were measured under the same conditions at a ligand concentration of $0.01 \mathrm{M}$.
NMR. All NMR measurements were performed with a Bruker Avance 500 spectrometer equipped with a $5 \mathrm{~mm}$ BBI-xyz-gradient probe. In Pulsed Field Gradient (PFG) ${ }^{1} \mathrm{H}-\mathrm{NMR}$ mode, the diffusion filtered spectra were recorded with the standard bipolar pulse sequence combining a radio-frequency stimulated spin-echo of the magnetization and a bipolar pulsed gradient sequence. The signal attenuation is given by the Stejskal-Tanner equation (Eq. 2):

$$
\frac{I}{I_{0}}=\exp \left(-\left(\gamma_{H} g \delta\right) D\left(\Delta-\frac{\delta}{3}-\frac{\tau}{2}\right)\right)
$$

where $I$ and $I_{0}$ are the signals at $g$ and zero gradient strength respectively, $\gamma_{H}$ is the hydrogen gyromagnetic ratio, $D$ is the diffusion coefficient of the considered species, $\delta$ and $\Delta$ are the gradient pulse duration and the delay during which the diffusion is observed and $\tau$ is the time interval between the bipolar gradient pulses. The diffusion filtered spectra were recorded with $\delta / 2=0.75 \mathrm{~ms}, \tau=0.5 \mathrm{~ms}$ and $\Delta=100 \mathrm{~ms}$. The amplitude of the trapezoidal gradient pulses was varied from $2 \%$ to $98 \%$ of the maximum amplitude $\left(48.2 \mathrm{G} . \mathrm{cm}^{-1}\right)$. The spectra were recorded in toluene- $d_{8}$ at 298 K. Data were analyzed with Win-NMR or Topspin Bruker softwares (Bruker Biospin, Wissembourg, France).

LESR. LESR experiments were carried out using an X-band ( $3 \mathrm{~cm}, 9.7 \mathrm{GHz})$ ER 200D SRC Bruker spectrometer with $100 \mathrm{kHz}$ field ac modulation for phase-lock detection. Samples were located in a Bruker ER 41040R optical transmission resonator (unloaded quality factor $\mathrm{Q}=7000$ ), illuminated at $473.3 \mathrm{~nm}$ with a $\mathrm{CW}$ output power of $22.1 \mathrm{~mW}$ by a laser module Oxxius 473L-20-COL-PP-LAS-01186 and cooled down to $20 \mathrm{~K}$ with a cryostat ESR 900

Samples for LESR were prepared on ITO-coated flexible polyethylene terephthalate (PET) substrates using the same solutions and spinning parameters as in the case of device fabrication (see below). They were inserted into ESR tubes after folding. Magnetic field intensities and frequencies have been separately measured to insure accurate $g$-values $( \pm 0.0002)$. When spectra are compared, they have been recorded in the same conditions (gain, modulation, accumulation times, accumulation number and microwave power). The microwave power dependence of the ESR signal of the different samples has been measured and all data presented have been acquired in non saturation regime.

Device fabrication. Devices with general structure ITO/PEDOT:PSS/ polymer: $\mathrm{PCBM}: \mathrm{NCs} / \mathrm{Al}$ were prepared under ambient conditions. The prepatterned ITO substrates were cleaned using acetone and ethanol in an ultrasonic bath for $10 \mathrm{~min}$ each. A layer of 30-40 nm PEDOT-PSS was spin-coated on the ITO surface and was dried at $200^{\circ} \mathrm{C}$ for $10 \mathrm{~min}$. Reference samples without nanoparticles were produced from ortho-dichlorobenzene (ODCB) solution containing $\mathrm{P} 3 \mathrm{HT}$ or PTAA and PCBM in equal weight ratio $(30 \mathrm{mg} / \mathrm{mL})$. In the case of ternary blends the appropriate amount of a $30 \mathrm{mg} / \mathrm{mL}$ colloidal solution of nanocrystals in ODCB was added to the binary mixture to obtain mass ratios of $1: 1: 1,1: 1: 0.5$ or $1: 1: 0.25$ (polymer: PCBM : NCs). In all cases, approximately $200 \mathrm{~nm}$ thick active layers were spin-coated on the ITO/PEDOT : PSS substrate by adjusting the spinning parameters, typically $800 \mathrm{rpm}$ during $180 \mathrm{~s}$. 80-100 $\mathrm{nm}$ top aluminium electrodes were thermally evaporated under vacuum (active area: $7 \mathrm{~mm}^{2}$ ). In last step, the devices were encapsulated with thin glass slides pasted with epoxy glue purchased from DELO Company.

Device characterization. Film thickness measurements were performed using stylus profilometer (Ambios Technology XP-2). For cross-section SEM imaging using a Zeiss Ultra-55 microscope, the samples were cleaved in liquid nitrogen before electrode evaporation.

For the external quantum efficiency measurements, the samples were illuminated with a mercury-xenon lamp through a Newport 74125 Cornerstone ${ }^{\mathrm{TM}} 260$ monochromator. Photocurrent measurements were performed with a Newport 70104 Merlin ${ }^{\mathrm{TM}}$ digital lock-in radiometry system.

I(V) measurements. Current-voltage characteristics and power conversion efficiencies of the solar cells were measured under inert atmosphere using a computer controlled Keithley 2400 unit and $1000 \mathrm{Wm}^{-2}$ air-mass 1.5 simulated solar light generated by a Newport class AAA solar simulator. A monocrystalline silicon solar cell, calibrated at Fraunhofer Institut für Solare Energiesysteme (Freiburg, Germany), was used as a reference cell.

Photo-CELIV. We used a home-built setup for Charge Extraction by Linearly Increased Voltage (CELIV) measurements equipped with a Crylas Nd:YAG laser emitting at $532 \mathrm{~nm}$ or $355 \mathrm{~nm}$, with a modulable output power up to $100 \mu \mathrm{J}$ in each $1 \mathrm{~ns}$ pulse. The laser beam was split into two secondary beams: one was sent to a photodiode that detects the laser pulse, and then triggered the generation of voltage pulses and the opening of the mechanical shutter once every measurement period. The other beam could be attenuated by optical densities before being focused into an optical fiber for transmission to the sample when the mechanical shutter was open. All measurements were performed in a Faraday cage and care has been taken to limit electrical noise. The sample holder was horizontal and allowed for illumination either through the bottom or the top electrode. Contacts were made by probes equipped with micro-manipulators. Voltage pulses and ramps were generated by an Agilent $33220 \mathrm{~A}$ waveform generator and a Trek $601 \mathrm{C}$ voltage amplifier if needed. Current was directly measured and recorded by a Tektronik digital oscilloscope DPO 7104 
$1 \mathrm{GHz}$ thanks to a $50 \Omega$ terminated probe and a current amplifier when needed. All electronics were synchronized on the laser pulse detection by a Stanford Research System delay generator DG535.

1. He, Z. et al. Enhanced power-conversion efficiency in polymer solar cells using an inverted device structure. Nat. Photon. 6, 591-595 (2012).

2. Janssen, R. A. J. \& Nelson, J. Factors Limiting Device Efficiency in Organic Photovoltaics. Adv. Mater. 25, 1847-1858 (2013).

3. Dimitrov, S. D. \& Durrant, J. R. Materials Design Considerations for Charge Generation in Organic Solar Cells. Chem. Mater. 26, 616-630 (2013).

4. Faist, M. A. et al. Competition between the Charge Transfer State and the Singlet States of Donor or Acceptor Limiting the Efficiency in Polymer:Fullerene Solar Cells. J. Am. Chem. Soc. 134, 685-692 (2011).

5. Silva, C. Organic photovoltaics: Some like it hot. Nat. Mater. 12, 5-6 (2013).

6. Yu, G., Gao, J., Hummelen, J. C., Wudl, F. \& Heeger, A. J. Polymer Photovoltaic Cells-Enhanced Efficiencies Via a Network of Internal Donor-Acceptor Heterojunctions. Science 270, 1789-1791 (1995).

7. Bansal, N. et al. Influence of Crystallinity and Energetics on Charge Separation in Polymer-Inorganic Nanocomposite Films for Solar Cells. Sci. Rep. 3, 1531 (2013).

8. Reiss, P., Couderc, E., De Girolamo, J. \& Pron, A. Conjugated polymers/ semiconductor nanocrystals hybrid materials-preparation, electrical transport properties and applications. Nanoscale 3, 446-489 (2011).

9. Saunders, B. R. \& Turner, M. L. Nanoparticle-polymer photovoltaic cells. Adv. Colloid Interface Sci. 138, 1-23 (2008).

10. Skompska, M. Hybrid conjugated polymer/semiconductor photovoltaic cells. Synth. Met. 160, 1-15 (2010).

11. Couderc, E. et al. Charge transport in poly(3-hexylthiophene):CdSe nanocrystals hybrid thin films investigated with time-of-flight measurements. Appl. Phys. Lett. 101, 133301 (2012).

12. Huynh, W. U., Dittmer, J. J. \& Alivisatos, A. P. Hybrid nanorod-polymer solar cells. Science 295, 2425-2427 (2002).

13. Ren, S. et al. Inorganic/Organic Hybrid Solar Cell: Bridging Quantum Dots to Conjugated Polymer Nanowires. Nano Lett. 11, 3998-4002 (2011).

14. Sun, B. Q. \& Greenham, N. C. Improved effciency of photovoltaics based on CdSe nanorods and poly(3-hexylthiophene) nanofibers. PCCP 8, 3557-3560 (2006).

15. Liao, H.-C. et al. Nanoparticle-Tuned Self-Organization of a Bulk Heterojunction Hybrid Solar Cell with Enhanced Performance. ACS Nano 6, 1657-1666 (2012).

16. Li, L. et al. Highly Luminescent $\mathrm{CuInS}_{2} / \mathrm{ZnS}$ Core/Shell Nanocrystals: CadmiumFree Quantum Dots for In Vivo Imaging. Chem. Mater. 21, 2422-2429 (2009).

17. Li, L. et al. Efficient Synthesis of Highly Luminescent Copper Indium SulfideBased Core/Shell Nanocrystals with Surprisingly Long-Lived Emission. J. Am. Chem. Soc. 133, 1176-1179 (2011).

18. Fritzinger, B. et al. In Situ Observation of Rapid Ligand Exchange in Colloidal Nanocrystal Suspensions Using Transfer NOE Nuclear Magnetic Resonance Spectroscopy. J. Am. Chem. Soc. 131, 3024-3032 (2009).

19. Moreels, I., Fritzinger, B., Martins, J. C. \& Hens, Z. Surface Chemistry of Colloidal PbSe Nanocrystals. J. Am. Chem. Soc. 130, 15081-15086 (2008).

20. Lefrançois, A. et al. Effect of the treatment with (di-)amines and dithiols on the spectroscopic, electrochemical and electrical properties of CdSe nanocrystals' thin films. J. Mater. Chem. 21, 11524-11531 (2011).

21. Cardona, C. M., Li, W., Kaifer, A. E., Stockdale, D. \& Bazan, G. C. Electrochemical Considerations for Determining Absolute Frontier Orbital Energy Levels of Conjugated Polymers for Solar Cell Applications. Adv. Mater. 23, 2367-2371 (2011).

22. Scharber, M. C. et al. Design rules for donors in bulk-heterojunction solar cells Towards 10\% energy-conversion efficiency. Adv. Mater. 18, 789-794 (2006).

23. MacDonald, B. I. et al. Layer-by-Layer Assembly of Sintered CdSe $\mathrm{Te}_{1-\mathrm{x}}$ Nanocrystal Solar Cells. ACS Nano 6, 5995-6004 (2012).

24. Heinemann, M. D. et al. Photo-induced Charge Transfer and Relaxation of Persistent Charge Carriers in Polymer/N anocrystal Composites for Applications in Hybrid Solar Cells. Adv. Funct. Mater. 19, 3788-3795 (2009).

25. Krinichnyi, V. I., Yudanova, E. I. \& Denisov, N. N. Light-induced EPR study of charge transfer in poly(3-hexylthiophene)/fullerene bulk heterojunction. J. Chem. Phys. 131, 044515-044526 (2009).

26. Ceuster, J. D., Goovaerts, E., Bouwen, A., Hummelen, J. C. \& Dyakonov, V. Highfrequency $(95 \mathrm{GHz})$ electron paramagnetic resonance study of the photoinduced charge transfer in conjugated polymer-fullerene composites. Phys. Rev. B 64, 195206-195212 (2001).

27. Pientka, M. et al. Photogeneration of charge carriers in blends of conjugated polymers and semiconducting nanoparticles. Thin Solid Films 451, 48-53 (2004).

28. Witt, F., Kruszynska, M., Borchert, H. \& Parisi, J. Charge Transfer Complexes in Organic/Inorganic Hybrid Blends for Photovoltaic Applications Investigated by Light-Induced Electron Spin Resonance Spectroscopy. J. Phys. Chem. Lett. 1, 2999-3003 (2010).

29. Abdou, M. S. A., Orfino, F. P., Son, Y. \& Holdcroft, S. Interaction of Oxygen with Conjugated Polymers: Charge Transfer Complex Formation with Poly(3alkylthiophenes). J. Am. Chem. Soc. 119, 4518-4524 (1997).

30. Reyes-Reyes, M., Kim, K. \& Carroll, D. L. High-efficiency photovoltaic devices based on annealed poly(3-hexylthiophene) and 1-(3-methoxycarbonyl)-propyl1-phenyl-(6,6)C-61 blends. Appl. Phys. Lett. 87, 083506 (2005).
31. Credgington, D., Hamilton, R., Atienzar, P., Nelson, J. \& Durrant, J. R. NonGeminate Recombination as the Primary Determinant of Open-Circuit Voltage in Polythiophene:Fullerene Blend Solar Cells: an Analysis of the Influence of Device Processing Conditions. Adv. Funct. Mater. 21, 2744-2753 (2011).

32. Li, G. et al. High-efficiency solution processable polymer photovoltaic cells by selforganization of polymer blends. Nat. Mater. 4, 864-868 (2005).

33. Kruszynska, M., Knipper, M., Kolny-Olesiak, J., Borchert, H. \& Parisi, J. Charge transfer in blends of P3HT and colloidally prepared CuInS2 nanocrystals. Thin Solid Films 519, 7374-7377 (2011).

34. Kim, Y. et al. A strong regioregularity effect in self-organizing conjugated polymer films and high-efficiency polythiophene:fullerene solar cells. Nat. Mater. 5, 197-203 (2006).

35. Pivrikas, A., Sariciftci, N. S., Juška, G. \& Österbacka, R. A review of charge transport and recombination in polymer/fullerene organic solar cells. Progr. Photovolt.: Res. Appl. 15, 677-696 (2007).

36. Mihailetchi, V. D., Xie, H. X., de Boer, B., Koster, L. J. A. \& Blom, P. W. M. Charge Transport and Photocurrent Generation in Poly(3-hexylthiophene): Methanofullerene Bulk-Heterojunction Solar Cells. Adv. Funct. Mater. 16, 699-708 (2006)

37. Bange, S., Schubert, M. \& Neher, D. Charge mobility determination by current extraction under linear increasing voltages: Case of nonequilibrium charges and field-dependent mobilities. Phys. Rev. B 81, 035209 (2010).

38. Mozer, A. J. et al. Charge carrier mobility in regioregular poly(3-hexylthiophene) probed by transient conductivity techniques: A comparative study. Phys. Rev. B 71, 035214 (2005).

39. Intaniwet, A. et al. Characterization of thick film poly(triarylamine) semiconductor diodes for direct X-ray detection. J. Appl. Phys. 106, 064513 (2009).

40. Choudhury, K. R., Winiarz, J. G., Samoc, M. \& Prasad, P. N. Charge carrier mobility in an organic-inorganic hybrid nanocomposite. Appl. Phys. Lett. 82, 406-408 (2003).

41. Chang, C.-H. et al. Improved charge separation and transport efficiency in poly (3hexylthiophene) $-\mathrm{TiO}_{2}$ nanorod bulk heterojunction solar cells. J. Mater. Chem. 18, 2201-2207 (2008).

42. Honda, S., Ohkita, H., Benten, H. \& Ito, S. Selective Dye Loading at the Heterojunction in Polymer/Fullerene Solar Cells. Adv. Energy Mater. 1, 588-598 (2011).

43. Lu, L., Xu, T., Chen, W., Landry, E. S. \& Yu, L. Ternary blend polymer solar cells with enhanced power conversion efficiency. Nat. Photon. 8, 716-722 (2014).

44. Huang, J.-S. et al. Polymer bulk heterojunction solar cells employing Förster resonance energy transfer. Nat. Photon. 7, 479-485 (2013).

45. Brinkmann, M., Aldakov, D. \& Chandezon, F. Fabrication of oriented and periodic hybrid nanostructures of regioregular poly(3-hexylthiophene) and CdSe nanocrystals by directional epitaxial solidification. Adv. Mater. 19, 3819-3823 (2007).

\section{Acknowledgments}

A.L. acknowledges financial support through the L'Oréal-Unesco Award for Women in Science. B.L., J.M.V. and P.R. thank CEA for funding via the Chimtronique 2015 program. J.F.V. acknowledges financial support from the French National Research Agency (MATISSE, Grant No. ANR-11-BS08-0001).

\section{Author contributions}

A.L. carried out the experiments with the help of B.L., B.B., J-M.V. (device fabrication), B.P.D., C.L. (LESR), M.G. (NMR), J.F.V. (mobility measurements), S.P. (XRD), F.C. (TEM) and S.S. (electrochemistry). P.R. conceived the study and wrote the manuscript. All authors contributed to discussions and manuscript review.

\section{Additional information}

Supplementary information accompanies this paper at http://www.nature.com/ scientificreports

Competing financial interests: The authors declare no competing financial interests.

How to cite this article: Lefrançois, A. et al. Enhanced Charge Separation in Ternary P3HT/ PCBM/CuInS 2 Nanocrystals Hybrid Solar Cells. Sci. Rep. 5, 7768; DOI:10.1038/srep07768 (2015).

This work is licensed under a Creative Commons Attribution-NonCommercialNoDerivs 4.0 International License. The images or other third party material in this article are included in the article's Creative Commons license, unless indicated otherwise in the credit line; if the material is not included under the Creative Commons license, users will need to obtain permission from the license holder in order to reproduce the material. To view a copy of this license, visit http:// creativecommons.org/licenses/by-nc-nd/4.0/ 